\title{
THE STRUCTURE OF MERGER REMNANTS OF COMPACT GROUPS OF GALAXIES: SOME PRELIMINARY RESULTS
}

\author{
E. ATHANASSOULA, CH. L. VOZIKIS \\ Observatoire de Marseille \\ 2, Place Le Verrier, 13248 Marseille Cedex 04, France
}

\begin{abstract}
We present some preliminary results on the properties of multiple merger remnants. We discuss their axial ratios, their $V / \sigma$ ratio as a function of ellipticity, and their circular velocity profiles.
\end{abstract}

\section{Introduction}

Since Toomre's pioneering suggestion that elliptical galaxies could be the result of a merger (Toomre \& Toomre 1972) a number of numerical simulations have focused on the properties of merger remnants of galaxy pairs (see e.g. the reviews of Barnes \& Hernquist 1992, or Barnes 1994 and references therein) in order to compare them with those of ellipticals. Although such comparisons show in general good agreement, they also put forward many points of disagreement, like the form of the radial density profile in the innermost parts, or the misalignment between the minor axis and the angular momentum vector. A variation to the original merging scenario is that elliptical galaxies are indeed merger remnants but not necessarily of pairs, but rather result from multiple mergers. In order to test this we are currently studying the properties of merger remnants of compact groups of five galaxies. Our work is complementary to that of Weil \& Hernquist (1996), who have more particles per simulation and therefore can examine features we cannot. However, we examine a larger variety of initial conditions and thus can study their effect on the structure of the merger remnant.

The simulations we discuss here have been presented by Athanassoula, Makino \& Bosma (1997). For the purposes of the present work we extended them in time to make sure that the remnants have reached equilibrium. In some of our simulations a common halo encompasses the whole group, while in others galaxies have individual halos. We have also used different values 
of halo-to-total mass, different density profiles for the distribution of mass in the group and in the common halo, and different initial kinematics of the group. Here we present only some preliminary results. Further results will be presented elsewhere (Vozikis \& Athanassoula, 1998).

\section{Axial ratios}

For all remnants of our simulations we calculated the axial ratios $b / a$ and $c / a$ for the luminous and dark material separately. For the luminous material we find average values $\langle c / a\rangle=0.7$ and $\langle b / a\rangle=0.9$. These values depend neither on whether the halo was common or individual, nor on the central concentration of the initial configuration. On the other hand the spread around the mean value is much bigger for simulations starting with individual halos than for those starting with common halos. For the dark material we find average values of $\langle c / a\rangle=0.85$ and $\langle b / a\rangle=0.95$, i.e. the dark matter is distributed in a more spherical manner than the luminous material. Simulations with common halos lead to remnants whose halos are more spherical than do simulations starting with individual halos. Simulations with a higher halo-to-total mass ratio give remnants with more spherical halos than simulations starting with lower halo-to-total mass ratios.

\section{3. $V / \sigma$ as a function of ellipticity}

Fig. 1 shows the position of the merger remnants evolving from simulations initially in virial equilibrium on the $V / \sigma$ as a function of ellipticity diagram. The values displayed were obtained by viewing the remnant perpendicular to the axis of rotation and taking a mass weighted average for all the material within the half mass radius. The area on this diagram occupied by the luminous material of the remnants is roughly the same as that occupied by big ellipticals (e.g. Davies 1987), although in the observational sample there are some faster rotators than we have in our simulations. The dark matter part of the remnants coming from simulations with initially individual halos covers a similar part of the diagram, due to the fact that the two components in such simulations have similar evolutionary histories. On the other hand the dark matter of remnants from simulations with initially common halos clusters in less elongated and less anisotropic regions, due to the fact that the initial common halos are spherical and isotropic.

Taking into account the values of the triaxiality parameter $T$ (Franx, Illingworth \& de Zeeuw 1991), as well as the values of the angle between the angular momentum vector and the minor axis, we find the following general trend for the luminous part of the remnants. Remnants with relatively high values of $V / \sigma$ (i.e. relatively fast rotators) are flatter, more oblate-like (i.e. 


\section{Luminous matter Dark matter}

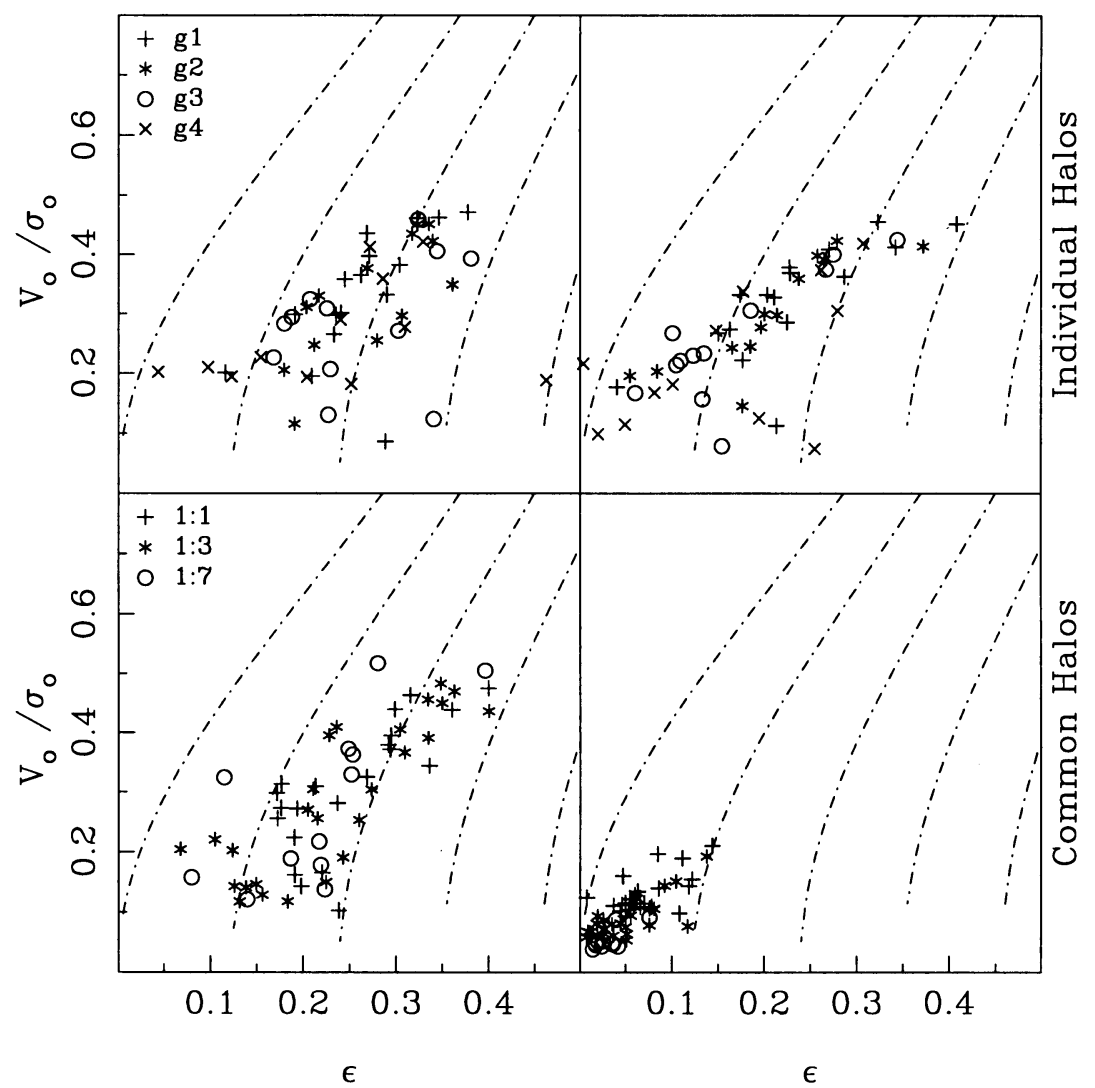

Figure 1. Location of the merger remnants from simulations initially in virial equilibrium on the $v / \sigma$ as a function of eccentricity diagram. The two left panels correspond to the luminous matter and the two right ones to the dark matter. The two upper panels refer to simulations with initially individual halos and the two lower ones to simulations with initially common halos. Different symbols correspond to different types of galaxies in the initial group (cf. Athanassoula, Makino \& Bosma (1997)), or different ratios of luminous to dark matter, as given in the figure. The dot-dashed lines correspond to the results for oblate spheroidals with values of the anisotropy parameter $\delta$ (from left to right) 0 ., 0.1 , $0.2,0.3$ and 0.4 .

have low values of the triaxiality parameter $T$ ) and show a good alignment between the angular momentum vector and the minor axis. On the other hand remnants which are slow rotators (i.e. have a relatively low values of $V / \sigma)$ are more spherical-like, have higher values of the triaxiality parameter and do not present a good alignment between the angular momentum vector and the minor axis. 


\section{Circular velocity profiles}

Assuming spherical symmetry, we can define the circular velocity profile from

$$
V_{\mathrm{c}}(r)=\sqrt{M(r) / r}
$$

where $M(r)$ is the total mass within the radius $r$. We have calculated this profile for all the merger remnants coming from our simulations and find that it depends on the initial conditions of the simulations. Thus remnants of groups with initially individual halos have circular velocity profiles that decrease fast with radius. Similar profiles can be found for cases where the dark matter was initially very centrally concentrated, or where it was a relatively small contribution to the total mass. On the other hand merger remnants from simulations with initially common halos, a relatively high halo-to-total mass ratio and an initial density profile which is not too centrally concentrated give circular velocity profiles which are rather flat or present two maxima, one near the center, due to the luminous material and the other quite far out, due to the dark matter. If these distinctions carry over to the rotation curves of these remnants then, by comparing them with observed rotation curves, we could get some information on the initial distribution of dark matter in groups and its relative amount.

Acknowledgments We thank A. Bosma for useful discussions and J.-C. Lambert for his help with the administration of the simulations. We also thank the INSU/CNRS and the University of Aix-Marseille I for funds to develop the necessary computing facilities. CV gratefully acknowledges an EEC TMR fellowship, which made this work possible.

\section{References}

Athanassoula, E., Bosma, A., Lambert, J.-C., Makino, J. (1997) Performance and accuracy of a GRAPE-3 system for collisionless N-body simulations, $M N R A S$, (in press), astro-ph/9707079.

Athanassoula, E., Makino, J., Bosma, A. (1997) Evolution of compact groups of galaxies I. Merging rates, MNRAS, 286, pp. 825-838.

Barnes, J. (1994) Interactions and mergers in galaxy formation, in The formation and evolution of galaxies, eds. C. Muñoz-Tuñón \& F. Sánchez, Cambridge univ. press, pp. 399-453.

Barnes, J. Hernquist, L. (1992) Dynamics of interacting galaxies, Ann. Rev. Astron. Astrophys., 30, pp. 705-742.

Davies, R. (1987) The stellar kinematics of elliptical galaxies, in Structure and dynamics of elliptical galaxies, ed. Tim de Zeeuw, Reidel Pub., pp. 63-77.

Franx M., Illingworth G.D. and de Zeeuw T. (1991) The ordered nature of elliptical galaxies: implications for their intrinsic angular momenta and shapes, $A p . J ., \mathbf{4 3 8}$, 112

Toomre A., Toomre J. (1972) Galactic bridges and tails, Ap. J., 178, pp 623-666.

Vozikis, Ch. L., Athanassoula, E. (1998), in preparation. 